\title{
CHROMOSOME STRUCTURE IN PERIPLANETA AMERICANA
}

\author{
B. JOHN \\ Genetics Department, Birmingham University \\ and \\ K. R. LEWIS \\ Botany Department, Oxford University
}

Received I I.xii. 59

IN general paraffin methods are inferior to squash techniques for chromosome preparations. From a study of preparations made with these older paraffin methods Sharma, Parshad and Sehgal (1956, I959) have criticised our conclusions regarding the organisation of the chromosomes in the American cockroach Periplaneta americana. In this paper we intend summarising the evidence which indicates that many of their observations are incorrect. In consequence we consider their arguments invalid.

Sharma et al. make the following claims :-

I. During the anaphase of mitosis and meiosis chromosome ends lead the way to the poles and all the centromeres are terminal.

2. The prophase of meiosis is cut short by the early dissolution of the nuclear membrane and the precocious activity of the division centres. As a consequence, chromosome replication occurs at pro-metaphase. On their view this means that there is insufficient time for chiasma formation and consequently diplotene-diakinesis is not represented in the meiotic sequence. They also conclude that homologues are held together, in the absence of chiasmata, "by the force of attraction due to the singleness of each homologue".

3. Colchicine treatment induces diplotene-diakinesis stages (John and Lewis, 1957) with chiasmata because it prolongs prophase.

Our own observations and conclusions are described below.

\section{CENTROMERE POSITION AND BEHAVIOUR}

(i) Chromosome morphology

Constrictions are visible in all the chromosomes at mitosis in untreated cells (plate, figs. I and 2) and they are particularly clear following colchicine treatment (plate, fig. 3). Many spindle inhibitors cause selective extension of chromosome constrictions (Sokolow, I939; Tjio and Levan, i950).

One of the largest chromosomes in the haploid complement has two constrictions, the one median, the other submedian (plate, fig. 4). We have already suggested that this may be the nucleolar organising chromosome (John and Lewis, 1959). The constrictions of the other 
chromosomes are median to subterminal in position. We consider these to be primary, centric, constrictions. On the view of Sharma et al that the centromeres are terminal, one would be forced to conclude that there are thirty-five secondary constrictions in the chromosome complement of male $P$. americana. This would surely be a record.

Interstitial constrictions are often visible in all the chromosomes at pachytene of meiosis and these also are extended by colchicine treatment (plate, figs. 7 and 8). Separation of the homologues begins at these constrictions and proceeds towards the chromosome ends (cf. figs. $a-d$, Lewis and John, 1957a). A similar behaviour is figured (Suomalainen, I946, plate II, figs. I I and I2) in untreated cells of Periplaneta australase. At prophase and pro-metaphase of the second meiotic division sister chromatids are also associated interstitially
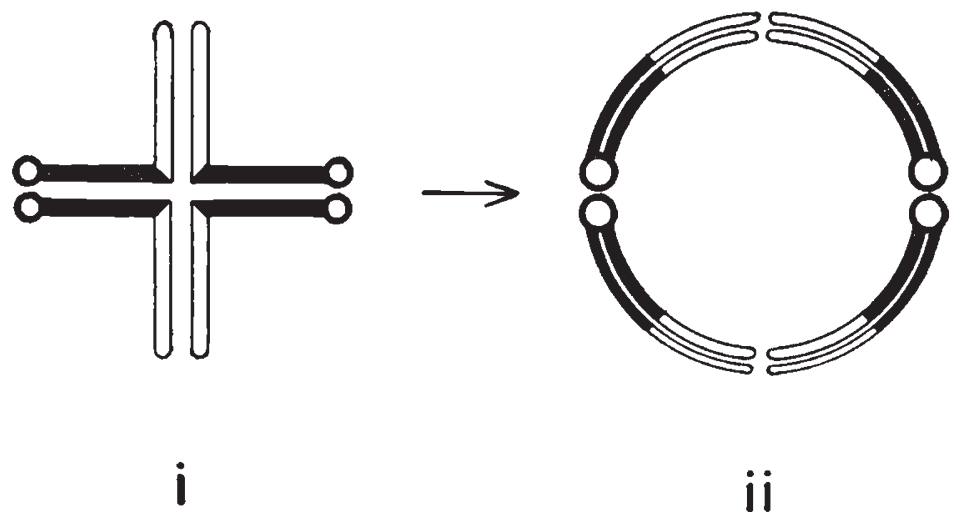

i

\section{ii}

TEXT-FIG. I.-Origin and nature of the interchange ring of four possible in an individual with telocentric chromosomes : (i) zygotene, (ii) pro-metaphase. Interstitial segments shown solid.

although the crosses so formed are somewhat deformed by matrical stickiness (plate, fig. 5).

All the evidence from chromosome morphology is thus against the conclusion that the centromeres are terminal. This evidence in itself does not of course disprove terminal kinetic activity, but it does prove that if there is such activity it is a secondary and not a primary event. Neo-centric activity of chromosome ends has been described, though only at meiosis (Prakken and Müntzing, 1942); but in P. americana the evidence from chromosome behaviour is as unequivocal as that from chromosome morphology in showing that the centromeres are not at the ends (plate, fig. 6). The observations of Suomalainen (1946, fig. 6r) are also in agreement with our findings.

\section{(ii) The behaviour of interchange rings}

The populations of $P$. americana which we have studied are characterised by having a high frequency of interchange heterozygotes (Lewis and John, 1957 $b$; John and Lewis, 1958). 
Where centromeres are terminal an interchange multiple of four with terminally associated members would have the structure shown in text-fig. I. This holds whether the maintenance of association is dependent on terminal chiasmata, terminal attraction independent of chiasma formation or both. This means that the multiple would be maintained by attraction between homologous centromeres even at metaphase. For Sharma et al. this poses no particular problem since on their view the formation of ring bivalents must depend on a similar attraction.

One of the factors affecting the fertility of interchange heterozygotes is the orientation of the multiples. A multiple must be arranged in a way such that adjacent centromeres move to opposite poles. The probability of disjunctional orientation is increased, especially for closed multiples, when the effective inter-centromeric distances are equal. In multiples with only terminal associations this condition is fulfilled when the chromosomes are equal in size and iso-brachial.

Multiples similar in many respects to the hypothetical example drawn in text-fig. I have been described in the short-horned grasshopper Trimerotropis citrina where the centromeres are very near the chromosome ends (Carothers, I93I). Theoretically, ring multiples could be formed in acrocentric types like this in two ways :-

(a) By the formation of chiasmata (or some other type of association) not only in both pairing segments but in both interstitial segments also. In this event centromeres would be absent from the closed loop. They would occur in half-loops which, because of their rotation, would lie at right angles to the closed loop. In this position homologous centromeres coorient but the presence of an association in the interstitial segments permits little or no interaction between nonhomologous centromeres, and the rigidity of the multiple means that adjacent non-homologous centromeres would often move to the same pole (see fig. 89, p. 264, Darlington, 1937). And this, in turn, means chromosome non-disjunction for those segments proximal to the interstitial chiasmata (and for all segments in the absence of chiasmata) together with chromatid non-disjunction for those segments distal to them. The multiples of this type described by Carothers (I93I) invariably behaved in this manner.

(b) A closed multiple could also be formed in a type with nearly terminal centromeres by the formation of chiasmata (or some other kind of association) in the pairing segments and in both short arms. In this case the centromeres would lie in the same plane and hence interaction could possibly occur. Where the second arms are very short, as they are in $\mathcal{T}$. citrina, a multiple of this type is not expected to occur often. Indeed it is difficult to ascertain from the early work of 
Carothers whether such a multiple was found. We think it probable, however, that the multiple in her fig. $35 \mathrm{f}$ is of this type and it was found only once. Whatever its exact structure, its orientation was non-disjunctional, like all the multiples described by Carothers irrespective of their form. We would conclude that this was so because homologous centromeres were too close together to co-orientate. In fact a multiple of this type is mechanically equivalent to a ring bivalent.

That centromeres can be too close for efficient co-orientation is indicated by the work of Revell (1947) and Rees (1955). The former found that in spiders, where the congression of polarised chromosomes is not in concert, bivalents with distally localised chiasmata congress first. Similarly in rye, Rees found that bivalents with proximally localised chiasmata were often delayed in their congression (see also Lewis, I958).

Where multiples are made up of chromosomes of unequal size centromeres are not equidistant and in many cases those which are close together move to the same pole even when this leads to numerical non-disjunction ( $c f$. fig. 52, p. I 52, Darlington, I937).

It is true that the pre-metaphase stretch, which in our opinion facilitates disjunction, is much more pronounced in $P$. americana than in short-horned grasshoppers. But it is very unlikely that the stretch would have much effect where the centromeres are terminal.

All these considerations indicate that the evolution of a system of permanent interchange hybridity, in a type with terminal or even nearly terminal centromeres, is so improbable that the possibility can be neglected. We trust that our investigations of some three hundred individuals from eleven populations of $P$. americana provide sufficient evidence that such a system obtains in the wild and cultured populations of this species in Great Britain. In this connection the populations studied by Sharma et al. would provide an interesting comparison with our coal mine populations. Far from being confined, the train compartment populations can move much more rapidly and farther than their own locomotory powers permit. This presumably leads to greater outbreeding. It is interesting to note, therefore, that Sharma et al. do not seem to have observed interchange multiples, though this too may be a result of the obvious handicap imposed by their consistent use of microtomy.

\section{CHROMOSOME REPLICATION}

There is no general agreement as to the time of DNA synthesis during the meiotic cycle and it appears to occur at slightly different times in different organisms (Moses and Taylor, I955; Taylor, 1959). Opinion is also divided with regard to the stage at which recombination occurs, but it is generally accepted that both replication and 
recombination are completed before diplotene. Even at this stage, however, the four chromatids and their chiasmate associations can often be resolved only in the most favourable material. This means that replication must occur during or before pachytene.

Because of the contracted state of the late pachytene bivalents (contra Sharma et al.) and their lampbrush appearance during the initial pre-stretch phase, we have found it impossible to resolve the chromatids at these stages in P. americana. Therefore we would ask Sharma $e t$ al. what observational evidence they have for concluding that replication in $P$. americana does not occur until pro-metaphase (Sharma, Parshad and Sehgal, I956). We find it difficult to believe that they have been able to resolve chromatids in preparations where the constrictions have remained undetected.

Their conclusion can then only be based on the evidence of the continued parallel pairing of homologues. Hughes-Schrader (I943) has, however, already shown that this situation obtains during the non-chiasmate meiosis of the male Callimantis after the chromosomes have replicated. Sharma et al. certainly comment on HughesSchrader's observation, but then proceed to ignore it by concluding that the "diplotene-diakinesis" suppression in P. americana can also be explained, like that in Callimantis, as due to the persistent singleness of each homologue throughout prophase. In fact they accept the suggestion made by White (I938) before Hughes-Schrader's (I943) observations were published. Even White himself no longer believes in this (White, I954, p. 23I).

There is no evidence to show or even suggest that chromosome replication in $P$. americana takes place at prometaphase and there is therefore no reason to entertain the suggestion made by Sharma $e t$ al. concerning the induction of diplotene-diakinesis by colchicine treatment. On the contrary, if the chromosomes are studied during the pre-metaphase stretch, especially in its early stages, it can be seen that the chromosomes are essentially similar in structure to those found in $c$-treated cells. They differ from them in only one respect, viz. their distortion, which is an inevitable consequence of the violent pre-metaphase movement. Colchicine treatment by inhibiting the spindle prevents this movement, but it does not produce a stage totally different from that found in untreated material. During the early stages of the pre-metaphase stretch the bivalents are lampbrush in appearance and the homologues are separated for the greater part of their length. The bivalents at induced diplotene are of the same basic form ( $c f$. plate, figs. 2 and 6, John and Lewis, 1957).

\section{BIVALENT ASSOCIATION}

Our earlier studies on $P$. americana led us to conclude that chiasmata are formed at least on occasion, though they must be localised near the chromosome ends in the majority of cases. Moreover matrical stickiness 
and terminal affinity may maintain chromosome association cven in the absence of chiasmata.

The strength of the latter forms of association may be judged from the consistent formation of terminal adhesions at first ana-telophase (plate, figs. 6, 9 and Io). Sharma et al. use these bridges to support their contention of precocious chromosome despiralisation. But it is clear from fig. 6 of the plate that the body of the chromosome is not despiralised at all; the bridges are the consequence of the persistent association of the ends of the chromosomes only.

Because chiasmata are clearly evident in some other cockroach species Sharma et al. argue that there is no reason why they should not be equally obvious in $P$. americana also. But there is every reason why they should be difficult to detect. Those factors which make it impossible to resolve chromatids, seriously interfere with the detection of chiasmata too. An even greater impediment, however, is the matrical stickiness and the occurrence of pre-metaphase stretch immediately after pachytene. As we have already pointed out, the bivalents at this time are not basically different from those at induced diplotenediakinesis. Particularly pertinent, therefore, is the fact that during the premetaphase stretch (plate, fig. I I), at metaphase (plate, fig. 9, Lewis and John, I957 $b$ ) and particularly at induced diplotene-diakineses (fig. e, Lewis and John, I $957 a$ ) subterminal associations can sometimes be seen.

In two other cockroach species where diplotene-diakinesis is a typical stage in the meiotic sequence, namely Leucophaa madere (Mathey, I95I) and Blaberus discoidalis (John and Lewis, I959) chiasmata can be satisfactorily resolved. In the former they terminalise quickly and in the latter they are confined to terminal euchromatic segments.

For the reasons given earlier it is not permissible in the absence of supporting evidence, to conclude that chromosome replication is delayed in those cockroaches where diplotene-diakinesis is absent. The minimum conclusion is that the pre-metaphase stretch, where it occurs immediately after pachytene, is an impediment to the observation of chiasmata; there is nothing to show that it interferes with their formation. The problem of resolving distally localised, rapidly terminalising chiasmata is by no means confined to cockroaches (John, 1957).

The situation is clarified further by the work of Callan and Jacobs (I957) on Mantis religiosa. The males of this mantid have a diploid complement of $24+\mathrm{X}_{1} \mathrm{X}_{2} \mathrm{Y}$ and, as in $P$. americana, there is a postpachytene stretch. The homologues remain parallely paired until the stretch and chiasmata cannot be distinguished, except for occasional subterminal associations. There are, however, two lines of evidence which in particular indicate that chiasmata are formed :

(a) Pairs of heterobrachial chromosomes may be associated by only their short arms. A force operative in the absence of chiasmata would be expected to separate short arms before long ones. 


\section{Plate}

Fic. 1.-Mitosis in a female blood cell (untreated, $\times 1600$ ).

Fig. 2.- - Male mitosis (untreated, $\times 1600$ ).

Fic. 3.-Male c-mitosis (Colchicine $2 \mathrm{hr}$., $\times 1600$ ).

Fig. 4.-Autosome with a secondary constriction (Colchicine $3 \mathrm{hr}$., $\times 3500$ ).

Fig. 5.-Pro-metaphase II (untreated, $\times$ I6oo).

Fig. 6.-Anaphase I (untreated, $\times 1600$ ).

Figs. 7 and 8.-C-pachytene in homozygous cells (Colchicine 4 hr., $\times$ Iooo).

Figs. 9. and I0.-Telophase I (untreated, $\times$ iooo).

Fig. I I.-Premetaphase stretch (R IV $+14 \mathrm{II}+\mathrm{X})$, note " chiasmate" bivalent (arrowed) and "lampbrush" nature of the autosomes (untreated, $\times 1000$ ). 
(b) Zygotene pairing is regular and complete; nevertheless univalents occur at metaphase. Univalent sex chromosomes are three times as common as unpaired autosomes though there are six times as many autosomes in the complement. What is more, $\mathrm{X}_{2}$ univalents are twice as common as $\mathrm{X}_{1}$ univalents. Both these observations can be readily explained on the assumption that metaphase association is dependent on chiasma formation : they then merely require the assumption that the pairing segments of the sex chromosomes are shorter than those of the autosomes and that of $\mathrm{X}_{2}$ is shorter than its counterpart in $\mathrm{X}_{1}$.

In summary, the organisation of the chromosomes in $P$. americana is not, as Sharma et al. intimate, purely a matter of opinion : it is a matter of observation and inference. We do not " appear to believe" that the centromeres are interstitial: we are convinced by observation that they are, and these observations leave no room for ambiguity. We are equally confident that colchicine-induced diplotene-diakinesis has its distorted counterpart in the normal meiotic sequence. Finally, we do not assume that chiasmata have become completely concealed by a prolongation of parallel pairing. On the contrary, we have seen and figured what in other organisms would be regarded as chiasmata. But we suggest that their terminal localisation together with matrical stickiness and the occurrence of the post-pachytene stretch makes it difficult to detect them consistently.

\section{REFERENCES}

CAllan, H. G., AND JACOBS, P. A. I957. The meiotic process in Mantis religiosa L. males. 7. Genet., 55, 200-2 17.

CAROTHERS, E. E. I93I. The maturation divisions and segregation of heteromorphic homologous chromosomes in Acridide (Orthoptera). Biol. Bull., 6I, 324-349.

Darlington, C. D. 1937. Recent advances in cytology. 2nd ed. Churchill, London. HUGHES-SCHRADER, s. I943. Meiosis without chiasmata in diploid and tetraploid spermatocyctes of the mantid Callimantis antillarum Saussure. F. Morph., 73, II I-I 4 I.

JoHN, в. 1957. The Chromosomes of zooparasites. II. Oswaldocruzia filiformis (Nematoda: Trichostrongylide). Chromosoma, 9, 6 1-68.

John, B., AND LewIS, K. R. 1957. Studies on Periplaneta americana. I. Experimental analysis of male meiosis. Heredity, II, I-9.

JOHN, B., AND LEWIS, K. R. 1958. Studies on Periplaneta americana. III. Selection for heterozygosity. Heredity, I2, 185-197.

JOHN, B., AND LEWIS, K. R. I 959 . Selection for interchange heterozygosity in an inbred culture of Blaberus discoidalis (Serville). Genetics, 44, 251-267.

LEWIS, K. R. 1958. Chromosome structure and organisation in Pellia epiphyla. Phyton, II, 29-37.

LEWIS, K. R., AND JOHN, в. 1957a. Bivalent-structure in Periplaneta americana. Nature, 179,973 .

LEWIS, K. R., AND JoHN, в. 1957b. Studies on Periplaneta americana. II. Interchange heterozygosity in isolated populations. Heredity, II, II-22.

D 2 
MATHEY, R. I95I. Systématique et critères cytologiques. Les chromosomes de Blanus cinereus Vand. (Lacertilia-Amphisbonida). et la méiose de Leucophaa maderce Brun. (Blattaria-Blaberidx). Bull. Soc. Vand. des Sci. Nat., 65, I I I-I 20.

MOSES, M. J., AND TAYLOR, J. H. I955. Deoxypentose nucleic acid synthesis during microsporogenesis in Tradescantia. Exp. Cell. Res., 9, 474-488.

PRAKKEN, R., AND MÜNTZING, A. I942. A meiotic peculiarity in rye, simulating a terminal centromere. Hereditas, 28, 442-482.

REES, H. I955. Genotypic control of chromosome behaviour in rye. I. Inbred lines. Heredity, 9, 93-1 I6.

ReVELL, S. H. I947. Controlled X-segregation at meiosis in Tegenaria. Hereaity, $I, 337-347$.

SHARMA, G. P., PARShAD, R., AND SEHGAL, P. 1956. Meiosis without chiasmata in Periplaneta americana. Nature, 178 , IOO4-IOO5.

SHARMA, G. P., PARSHAD, R., AND SEHGAL, P. 1959. Cytological analysis of the male meiosis in Periplaneta americana. 7. Genet., 56, 28I-287.

sokolow, I. 1939. Einfluss des Colchicins auf die Spermatogonial mitosen bei den Orthopteren. C.R. Acad. U.R.S.S., 24, 298-300.

suomalainen, E. 1946. Die Chromosomenverhältnisse in der Spermatogenese einiger Blattarien. Ann. Acad. Sci. Fenn., A, 4, Io, I-6o.

TAYLOR, J. H. 1959. Autoradiograph studies of nucleic acids and proteins during meiosis in Lilium longiforum. Amer. 7. Bot., 46, 477-484.

TJIO, I. H., AND LEVAN, A. I950. Quadruple structure of the centromere. Nature, I65, 368 .

White, M. J. D. 1938. A new and anomalous type of meiosis in a mantid, Callimantis antillarum Saussure. Proc. Roy. Soc., B. 125, 516-523.

white, м. J. D. 1954. Animal cytology and evolution. 2nd ed. Univ. Press, Cambridge. 